Check for updates

Cite this: Nanoscale Horiz., 2021, 6,839

DOI: $10.1039 / \mathrm{d} 1 \mathrm{nh} 90041 \mathrm{~b}$

rsc.li/nanoscale-horizons

\section{Horizons Community Board collection: solar energy conversion}

\author{
Rebecca L. M. Gieseking, (D) *a Alexandra J. Ramadan (D) ${ }^{b}$ and Jungki Ryu (D) *cd
}

Materials Horizons and Nanoscale Horizons set up their Community Boards several years ago, aiming to support early career researchers so that they can share their experiences and ideas on scientific publishing. As future leaders in their

\footnotetext{
${ }^{a}$ Department of Chemistry, Brandeis University, 415 South Street, Waltham, Massachusetts, 02453, USA.E-mail: gieseking@brandeis.edu

${ }^{b}$ Clarendon Laboratory, Department of Physics, University of Oxford, Oxford, OX1 3PU, UK. E-mail: alexandra.ramadan@physics.ox.ac.uk ${ }^{c}$ Department of Energy Engineering, School of Energy and Chemical Engineering, Ulsan National Institute of Science and Technology (UNIST), Ulsan, 44919, Republic of Korea. E-mail: jryu@unist.ac.kr

${ }^{d}$ Emergent Hydrogen Technology R\&D Center, Ulsan National Institute of Science and Technology (UNIST), Ulsan, 44919, Republic of Korea
}

respective fields, the Community Boards also provide a channel for members to build relationships across their research communites and develop their own editorial skills.

This collection continues a series of post-publication online article collections, led by our Community Board members across both Materials Horizons and Nanoscale Horizons.

Working together and sharing their unique areas of expertise, our Community Board members have recommended several key topics where significant, rapid progress has been made in the last 2 years. They have selected top articles published in the Horizons journals to showcase the most important advances in each topic area.

\section{Solar energy conversion}

Rebecca Gieseking, Alexandra Ramadan and Jungki Ryu present this Horizons Community Board collection on the fundamental understanding of various solar energy conversion technologies and devices.

The efficient conversion of solar energy to various forms has long been an ultimate goal of scientists and engineers to realize a sustainable and carbon-neutral society. Two of the most important solar energy conversion technologies are photovoltaics, which convert solar energy into electrical energy, and photocatalysts, which use solar energy to induce chemical reactions, often to produce fuels. These two approaches to harnessing solar energy share many

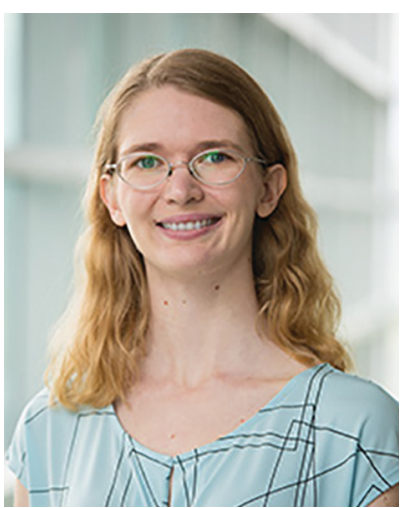

Rebecca L. M. Gieseking
Materials Horizons Community Board member Rebecca Gieseking earned her PhD in Chemistry from Georgia Tech in 2015. After three years as a postdoctoral fellow at Northwestern University, she started her independent career as an Assistant Professor at Brandeis University in 2018. Her research interests focus on developing lowcost quantum mechanical models to understand electron transfer processes in materials for emerging photochemical and electrochemical energy technologies.

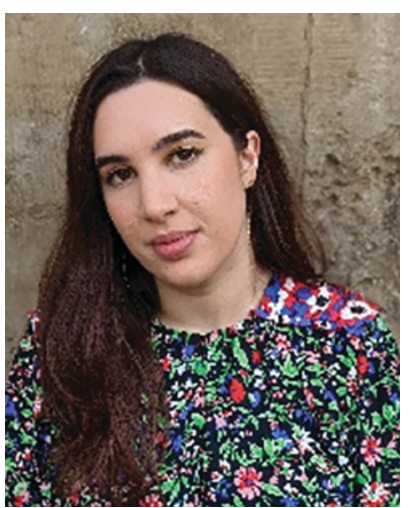

Alexandra J. Ramadan
Materials Horizons Community Board member Dr Alexandra Ramadan received her $P h D$ from the Department of Physics at Imperial College London in 2016. She has since worked as a postdoctoral researcher at the University of Oxford. In 2017 she was awarded a UK-US Fulbright Scholarship to work at Princeton University as a visiting researcher. Her main research interests are metal halide perovskites and organic semiconductors for photovoltaic and light emitting diode applications. 
common features, from fundamental photophysical and photochemical processes to device architectures to areas of ongoing challenges and development. As a result, they are complementary approaches to effectively use the practically unlimited but intermittent solar energy. In this themed collection, we have compiled articles that address fundamental issues and current challenges in photovoltaics and photocatalysis that provide insight into the development of innovative solar energy conversion devices.

\section{Photovoltaics}

Although there are many classes of materials used to create photovoltaic cells, the two classes with the most ongoing materials research are organic materials and metal halide perovskites. Organic photovoltaics have long been of interest because they have the potential to be manufactured at low costs and can be made into flexible devices. Because of the breadth of chemical space, computational modeling and machine learning techniques are powerful tools to give insight into which chemical structures promote isotropic charge transport (Mondelli et al., DOI: 10.1039/c9mh01439j), to rapidly screen donor-acceptor pairs for high photovoltaic efficiency (Padula et al., DOI: 10.1039/C8MH01135D) and to inform design principles for low disorder molecular semiconductors (Nematiaram et al., DOI: 10.1039/D0MH01159B). Spectroscopic tools that give insight into the charge separation process (Kang et al., DOI: 10.1039/D0MH01810D) can also provide fundamental understanding to develop the next generation of organic photovoltaics.

Photovoltaics based on metal halide perovskite semiconductors have seen a meteoric rise in efficiency over the past decade. A large part of the growth in their performance has been a result of the ability to produce high-efficiency devices through facile solution processing routes.

A range of solvent systems have been reported for the solution processing of metal halide perovskites, but with concerns over the toxicity of some of these solvents, research efforts have grown in developing green solvent systems (Wang et al., DOI: 10.1039/C9MH01679A) which can produce devices with equally impressive performances. Understanding the role of defects and charge traps in these materials is essential for the continued development of technologies based on these materials. A review on traps from Jin et al. (DOI: $10.1039 / \mathrm{C} 9 \mathrm{MH} 00500 \mathrm{E}$ ) discusses the origins of defects in metal halide perovskites and reviews methods adopted to mitigate their impact on device performances. One approach widely implemented within the field is the use of additives within the precursor solution to improve the crystallinity and morphology of metal halide perovskites (Lyu et al., DOI: 10.1039/D0NH00263A). In addition to their impressive performance in single junction photovoltaics, the tunable band gaps of metal halide perovskite semiconductors make them particularly attractive as the top cell in crystalline Si-based tandem architectures. The success of combining these two technologies together in efficient tandem devices hinges

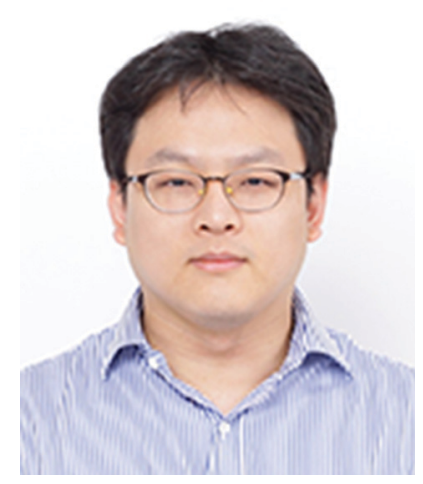

Jungki Ryu
Nanoscale Horizons Community Board member Jungki Ryu is an Associate Professor in the School of Energy and Chemical Engineering at the Ulsan National Institute of Science and Technology (UNIST). He received his BS and $\mathrm{PhD}$ in Materials Science and Engineering from Yonsei University in 2006 and the Korea Advanced Institute of Science and Technology (KAIST) in 2011. Before joining UNIST in 2014, he had studied bioinspired functional materials as a postdoctoral associate at the Massachusetts Institute of Technology for 3 years. His current research interests include electrocatalysis, solar fuel production, biomass utilization, and electrochemical waste refinery. on their electronic coupling through a recombination junction. The principles, properties and prospects of these junctions are discussed in a review by De Bastiani et al. (DOI: 10.1039/C9MH00500E).

\section{Photocatalysts}

Photocatalysts allow the green production of various chemicals, such as hydrogen, hydrocarbons/alcohols, and organic compounds, through a series of photophysical and photo(electro)chemical processes. Thus, a comprehensive understanding of light-matter interactions, charge carrier dynamics, and electrochemical reactions is required to design more efficient photocatalysts. A simple but highly effective way to improve the light-harvesting efficiency of photocatalysts is to design more efficient light absorbers. Wang et al. (DOI: 10.1039/ C9MH00257J) reported the design and fabrication of quadruple-band InGaN nanowires for broadband photocatalytic overall water splitting. Byun et al. reviewed recent works on conjugated porous polymers as an emerging class of photocatalysts (DOI: 10.1039/C9MH01071H). Lim et al. reviewed the broad spectrum of materials that can catalyze the desulfurization of fuels (DOI: 10.1039/D1NH00127B). Solar irradiation can also be more effectively captured via diverse forms of light-matter interactions. Plasmonic nanostructures are an emerging class of photocatalysts because of their exceptionally strong absorption. Tatsuma et al. reviewed recent work showing that plasmonic hot holes can be harnessed for photocatalysis (DOI: 10.1039/C9NH00649D). $\mathrm{Fu}$ et al. showed that plasmonic nanocrystals can be incorporated into 3D printable photocatalytic devices (DOI: 10.1039/D0MH01275K). Even though it comprises a large portion of solar irradiation, infrared light has rarely been utilized in photocatalysis because of its low energy. Tian et al. (DOI: 10.1039/C8NH00154E) summarized recent studies on near infrared light-active upconversion materials for full-spectrum photocatalytic systems.

Extending the lifetimes of charge carriers is critical to improving the efficiency of many solar technologies. Introducing heterojunction structures is an efficient way to extend those lifetimes. Sulas-Kern et al. 
(DOI: $10.1039 / \mathrm{C} 9 \mathrm{MH}$ 00954J) and Selopal et al. (10.1039/C8NH00227D) reported that charge carriers have prolonged lifetimes when transition metal dichalcogenides and quantum-dot-sensitized $\mathrm{TiO}_{2}$ are hybridized with carbon nanotubes, respectively. Zhao et al. (DOI: 10.1039/D0MH00955E) showed that carbonized polymer dots can enhance charge separation in lead halide perovskites for photocatalytic hydrogen production. Lin et al. (DOI: 10.1039/D0NH00491J) suggested a new concept of a surface domain heterojunction structure for efficient charge separation in rutile $\mathrm{TiO}_{2}$ photocatalysts. A review article by $\mathrm{Li}$ et al. (DOI: 10.1039/ D0NH00219D) summarized recent studies about the implementation of ferroelectric materials in photocatalysts for efficient charge separation.
Cocatalysts are key components for the efficient and selective photocatalytic production of target chemicals using separated charge carriers. Wang et al. showed that $\mathrm{Cu}_{2} \mathrm{~S}$ monolayers are efficient photocatalysts for $\mathrm{CO}_{2}$ reduction to ethanol (DOI: 10.1039/D1NH00196E). Kim et al. reported that a $\mathrm{Zn}$-based polyoxometalate can be employed as a $\mathrm{CO}_{2}$ reduction catalyst for the selective photocatalytic production of $\mathrm{CH}_{4}$ (10.1039/D0NH00657B), whereas $\mathrm{Zn}$ or $\mathrm{Zn}$-based materials are conventionally known as CO producing catalysts. Crocker et al. developed enzymeinspired photocatalysts by incorporating flavin into polydopamine for biomedical applications (10.1039/C9NH00199A). Handoko et al. (DOI: 10.1039/c9nh00100j) reviewed the application of two-dimensional MXenes as cocatalysts for various photocatalytic reactions.

These studies show that improving the efficiency of solar energy capture in its various forms is based on work at a broad range of length scales, from a fundamental understanding of atomistic material properties to nanoscale material architecture to device structures. Although photovoltaic and photocatalytic devices have important differences in their materials and operating principles, there is also much insight that can be gained by examining the similarities in their photophysics. We hope this themed collection will inspire new efforts to harness solar energy in these and other technologies. 